\title{
Jerry D. Tersoff to receive 2019 Von Hippel Award for advancing the understanding of low-dimensional and nanoscale electronic materials
}

$\mathrm{T}^{\mathrm{n}}$ 2019 Von Hippel Award, the Materials Research Society's (MRS) highest honor, will be presented to Jerry D. Tersoff of the IBM T.J. Watson Research Center. Tersoff is being recognized "for advancing the understanding of low-dimensional and nanoscale electronic materials, surfaces and interfaces, through elegant theoretical models that highlight the essential physics controlling growth, structure and electronic properties." Tersoff will present his award talk at the 2019 MRS Fall Meeting in Boston on Wednesday, December 4, at 5:45 pm, in the Grand Ballroom of the Sheraton Boston Hotel.

Tersoff is a principal research staff member at the IBM T.J. Watson Research Center. His recent research includes semiconductor nanowire growth; nanoscale effects in heteroepitaxy, including stressdriven self-assembly and self-organization; and the physics of carbon nanotube devices. His early work includes theories of scanning tunneling microscopy, Schottky barriers, and heterojunction band lineups.
He also developed model interatomic potentials that are widely used in materials simulations. His work has been recognized by the Davisson-Germer Prize of the American Physical Society (APS), the Medard W. Welch Award of the American Vacuum Society (AVS), and the MRS Medal. Tersoff is a member of the National Academy of Engineering and a Fellow of the APS, AVS, and MRS. He served on the MRS Board of Directors from 2003 to 2005 . He received his BA degree in physics from Swarthmore College and his $\mathrm{PhD}$ degree in physics from the University of California, Berkeley.

His presentation will focus on "Simple Models for Complex Behavior in Nanowire Growth." Semiconductor nanowires provide unique opportunities for studying crystal growth. Their small diameter guarantees single-crystal growth. He will describe some of his observations and show how even simple models can provide useful insights into behavior, thus deepening the understanding of nanoscale materials.

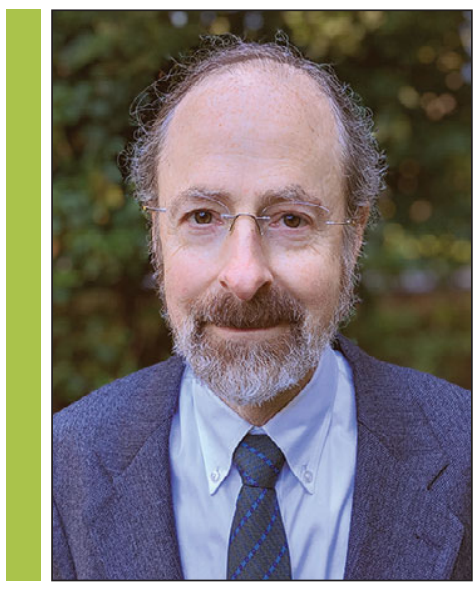

Named after Arthur von Hippel (18982003), the award recognizes the qualities most prized by materials scientists and engineers - brilliance and originality of intellect, combined with a vision that transcends the boundaries of conventional disciplines, as exemplified by the life of Arthur von Hippel (mrs.org/vonhippel).

The MRS Von Hippel Award includes a $\$ 10,000$ cash prize, honorary lifetime membership in MRS, and a trophy.

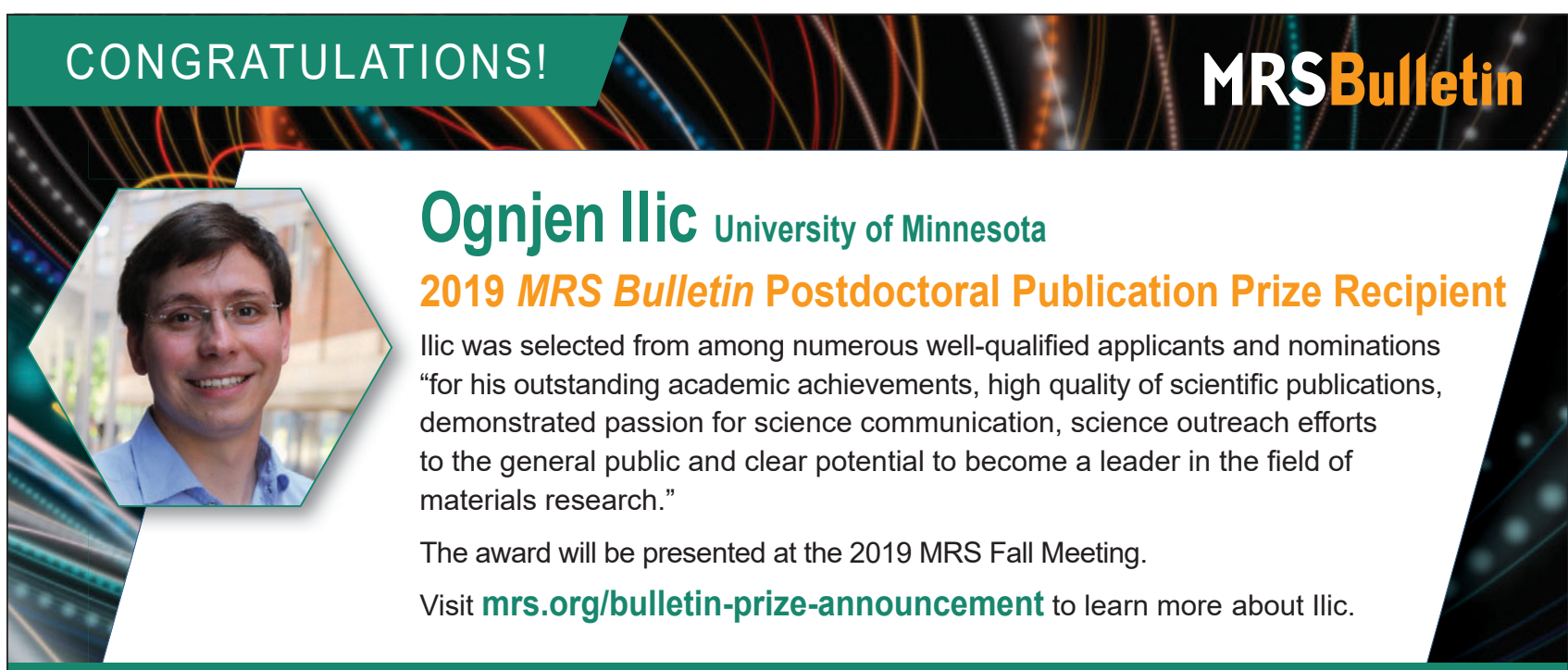

MRS Bulletin acknowledges the Jiang Family Foundation and MTI Corporation for their generous contributions to support this Prize. 